\title{
CHOQUE CULTURAL EN LAS AULAS: PROFESORES ANALÓGICOS vS ALUMNADO DIGITAL. EL CASO DE ANA.
}

\section{CULTURE SHOCK IN THE CLASSROOM: ANALOG TEACHERS vS DIGITAL STUDENTS. THE CASE OF ANA.}

\author{
Blas Segovia Aguilar; bsegovia@uco.es \\ Rosario Mérida Serrano; ed1meser@uco.es \\ Elena González Alfaya; ed2goalm@uco.es \\ Ma Ángeles Olivares García; ed1olgam@uco.es \\ Universidad de Córdoba
}

\section{RESUMEN}

En este artículo realizamos un estudio de caso con la pretensión de analizar e interpretar los factores que configuran la confrontación que se produce en los escenarios escolares entre la cultura tecnológica de educadores y la de los escolares. Abordamos la investigación desde una perspectiva cualitativa, con la finalidad de profundizar en los significados que los diversos agentes educativos atribuyen al uso de las TIC en el contexto familiar y en el escolar.

PALABRAS CLAVE: Educación, alfabetización digital, competencia digital, educación mediática, brecha digital, estudio cualitativo.

\section{ABSTRACT}

In this paper we describe a case study carried out in order to analyse and interpret the factors which shape the confrontation witnessed in school settings between the technological culture of educators and that of the students. In this investigation we follow a qualitative approach aimed at understanding the meanings attributed by different education agents to the use of ICT in the family and school context.

KEYWORDS: Education, digital literacy, digital qualification, media education, digital divide, qualitative research. 


\section{INTRODUCCIÓN}

El estudio que presentamos en este artículo se encuadra dentro de las investigaciones que indagan sobre las prácticas de uso de las tecnologías de la información y de la comunicación (TIC) en los centros y aulas.

Deriva del proyecto de investigación Escenarios, Tecnologías Digitales y Juventud en Andalucía $^{1}$, y analiza las diferentes culturas que conviven en el aula de un centro de secundaria respecto a los usos y apropiaciones de las TIC por parte de alumnado y del profesorado. El eje central del trabajo es el estudio de caso realizado sobre Ana, una alumna de 4 9 de ESO de un instituto público de Córdoba capital, centrándonos en los significados que atribuye al uso de las TIC en diversos ámbitos: el familiar, el escolar y los espacios de ocio. En este trabajo focalizamos nuestra atención sobre las diversas culturas -la del alumnado y profesorado- que conviven en el aula de un instituto que ha participado en el Programa de Centros TIC de Andalucía desde el curso 2005-2006, continuando en la actualidad con la implementación del Proyecto Escuela TIC 2.0.

\section{La escuela como escenario de confrontación digital}

Los cambios provocados por la Sociedad Informacional en las dinámicas sociales están configurando una nueva cultura que afecta a la escuela, pues en ella conviven personas interactuando con las tecnologías de forma diferente. Por un lado, el profesorado que adopta unas herramientas a instancias de los programas institucionales en las que no ha sido socializado, y, por otro, el alumnado, perteneciente a la generación net, que ha crecido en un entorno digital apropiándose de las tecnologías de forma natural.

Por eso, no es de extrañar que se produzcan tensiones entre los representantes de la cultura escolar y los de la cultura popular, sobre todo en aspectos relacionados con la gestión de la información y las prácticas que realizan cuando incorporan las modernas tecnologías a los procesos de aprendizaje. A veces olvidamos, que las concepciones del profesorado descansan en las ideas y experiencias en las que ha sido formado, y la mayoría de ellos lo ha sido en la cultura letrada (Lankshear \& Novell, 2008). Sin embargo, en el momento actual es necesario redefinir su papel en un entorno altamente mediatizado por las TIC, en el que el acceso a la información y al conocimiento se gestiona bajo otros parámetros. Una de las conclusiones destacables en los estudios realizados sobre el impacto de las TIC en las prácticas escolares es que a pesar del incremento en las dotaciones tecnológicas en las escuelas (hardware y software), la práctica pedagógica de los docentes en el aula no supone necesariamente una alteración sustantiva del modelo de enseñanza tradicional, por lo que el profesorado utiliza las tecnologías para apoyar pedagogías existentes (Balanskat, Blamire \& Kefala, 2006; Condie \& Munro, 2007; Coll, 2008; Area, 2010; Sigalés, Mominó \& Meneses, 2010).

Muchas de estas prácticas escolares reproducen el mismo modelo de aprendizaje de la escuela predigital, a través de modelos comunicativos que mantienen estructuras jerárquicas en los que el profesor dosifica y administra el discurso, apoyándose en el de los libros de texto o en el de una página web o favoreciendo actividades en las que la búsqueda de la información es el principal objetivo (Area, 2011).

\footnotetext{
${ }^{1}$ Proyecto de Excelencia (Ref. HUM-02599), financiado por la Junta de Andalucía, Consejería de Innovación Ciencia y Empresa.
} 


\section{MÉTODO}

Presentamos una aproximación cualitativa (Denzin \& Licoln, 1994) centrada en el análisis en profundidad de una unidad social particular con el fin de conocer y comprender el uso de las TIC en dos contextos -el académico y el familiar- regidos tradicionalmente por normas, valores, tradiciones y modos de acción diferentes. Desde una perspectiva ecológica y sistémica (Eisner, 1998) necesitamos comprender las discrepancias y continuidades que se producen en dos escenarios que interactúan, y en los que la adolescencia convive diariamente, constituyendo referencias ineludibles para sus modos de actuar en los escenarios digitales, así como para la construcción de nuevos aprendizajes y de su identidad digital.

Utilizamos un estudio de caso (Coller, 2000) de carácter cualitativo y exploratorio, para conocer la realidad social de un sujeto -Ana- y, a partir de dicho conocimiento realizar conclusiones válidas que ilustren la problemática planteada. Según Stake (1998: 11) es el "estudio de la particularidad y de la complejidad de un caso singular, para llegar a comprender su actividad en circunstancias importantes".

Para la selección de Ana, considerada como objeto de estudio de naturaleza social, empleamos los criterios de relevancia y pertinencia (Huberman, 1998). Buscamos un caso típico de contexto familiar con alta densidad tecnológica, del que forme parte una adolescente con un modo típico de uso de las TIC. El caso seleccionado por su normalidad, o repetida presencia social, contiene unas características similares a otros adolescentes de clase media que viven en la ciudad de Córdoba, lo cual facilita la transferibilidad de nuestros hallazgos. Su voluntariedad y disponibilidad para participar en el estudio son decisivos para la elección, al convertirla en una buena informante clave (Clandinin \& Connelly, 2000).

Los interrogantes que focalizan nuestra indagación responden a un proceso emergente (Van Dijk, 2001). Se han ido reformulado en el transcurso de la investigación en función del discurso que los participantes van construyendo. Los más relevantes son:

- ¿Qué impacto tienen las TIC en la transformación de las prácticas educativas?

- ¿Qué actitudes considera el profesorado de Secundaria que tienen los docentes de esta etapa ante las TIC?

- ¿Cuál es la percepción del alumnado sobre la competencia digital de los docentes?

- ¿Cuál es la percepción del profesorado sobre la competencia digital del alumnado de Educación Secundaria?

Las personas que participan en el estudio de caso de Ana son su madre y hermana mayor, en el contexto familiar, y dos profesores del centro, uno que desempeña el cargo de subdirector y el otro que se encarga de coordinar el Proyecto TIC del centro.

Para la recogida de información de este caso utilizamos la entrevista semiestructurada, la observación-participante, la conversación y el análisis documental (textual y fotográfico). Los contextos e instrumentos empleados para acceder a la información son:

- Entrevista y observación participante con la adolescente, su madre y hermana mayor en el hogar familiar.

- Entrevista y observación participante con Ana en su hogar familiar. 
- Conversación con la adolescente en un cibercafé.

- Entrevista al profesor-coordinador del Plan TIC del Instituto, realizada en el centro.

- Entrevista al subdirector del Instituto, realizada en el centro.

- Análisis documental de la página web y del Proyecto TIC del Instituto.

- Análisis de material gráfico: pantallazos del ordenador de Ana y fotografías.

\begin{tabular}{|c|c|}
\hline DIMENSIONES & CATEGORÍAS \\
\hline Densidad tecnológica & $\begin{array}{l}\text { Cat. 1: Tipos de tecnología } \\
\text { Cat. 2: Lugares }\end{array}$ \\
\hline Interacción tecnológica & $\begin{array}{l}\text { Cat. 1: Acceso } \\
\text { Cat. 2: Propiedad } \\
\text { Cat. 3: Usos } \\
\text { Cat. 4: Transgresiones } \\
\text { Cat. 5: Autonomía }\end{array}$ \\
\hline Interacción en la red & 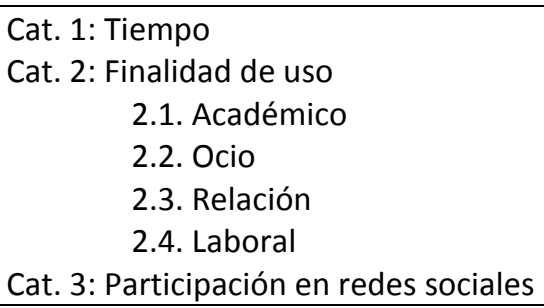 \\
\hline Percepción de los adultos & $\begin{array}{l}\text { Cat. 1: Temores } \\
\text { Cat. 2: Control y exigencias } \\
\text { Cat. 3: Visión según género }\end{array}$ \\
\hline Percepción adolescentes & $\begin{array}{l}\text { Cat. 1: Temores } \\
\text { Cat. 2: Control y exigencias } \\
\text { Cat. 3: Visión según género }\end{array}$ \\
\hline Competencia digital & $\begin{array}{l}\text { Cat. 1: Acceder } \\
\text { Cat. 2: Buscar } \\
\text { Cat. 3: Seleccionar } \\
\text { Cat. 4: Evaluar } \\
\text { Cat. 5: Comunicar }\end{array}$ \\
\hline
\end{tabular}

Tabla 1: Dimensiones y categorías de análisis

Los datos recabados de carácter textual provienen de las entrevistas que han sido realizadas, tanto en el contexto familiar como en el escolar. Estos datos han sido transcritos y analizados siguiendo la técnica de análisis del discurso (Bolívar, 2007). Se aplica un procedimiento artesanal (Farías \& Montero, 2005) para establecer distintas unidades de análisis. En primer lugar, se fijan las dimensiones o unidades de análisis macro para delimitar los ámbitos temáticos más relevantes. En una segunda fase se identifican las unidades de análisis micro, las categorías, incluidas en cada dimensión de las establecidas previamente. A continuación, se realiza una codificación de cada una de ellas. En todo el proceso descrito hemos desarrollado un acuerdo interjueces -cinco expertos participantes en la investigación señalada-, ya que las personas implicadas han realizado una propuesta personal, la cual ha sido contrastada con las del resto del equipo, incorporando las categorías coincidentes y desestimando las discrepantes. El sistema de categorías definitivamente establecido ha sido el esquema analítico aplicado para comprender y desentrañar los significados de los discursos expresados por los participantes. En la tabla 1 se presenta el sistema categorial, referido a ambos escenarios, del que para este trabajo usamos solo una parte. 


\section{RESULTADOS}

\subsection{Cartografía personal de Ana}

Ana es la tercera hija de un matrimonio de clase media-baja que vive en una casa de un barrio situado en la periferia de la ciudad de Córdoba. Es una alumna de 16 años que se considera alegre, sincera, cariñosa y curiosa. Repite 4ㅇ de ESO y no muestra mucho interés por los estudios.

Contrasta su desinterés por los estudios con su participación en actividades preprofesionales como la asistencia a una clínica veterinaria para hacer prácticas o su dedicación al mundo de la moda, colaborando en una agencia de modelos.

Respecto a sus aficiones destaca el deporte, concretamente, practica voleibol y también le gusta el mundo de la pasarela. Su tiempo de ocio, además, lo dedica al uso de las TIC, sobre todo el ordenador y el móvil. Lo que más le gusta es entrar en la red social Tuenti, aunque en los últimos tiempos también utiliza con asiduidad el Messenger. El móvil lo emplea básicamente para enviar mensajes y llamar a su familia.

\subsection{Ecosistema tecnológico del contexto familiar}

En el domicilio familiar de Ana podemos apreciar una densidad tecnológica media-alta, si tomamos como referencia los resultados del estudio realizado por el Instituto Nacional de Tecnologías de la Comunicación (2009: 35), donde se indica que "la densidad tecnológica media de los hogares españoles es de 1.3 ordenadores por hogar, siendo la presencia del ordenador personal de sobremesa cinco veces superior al ordenador portátil".

En la vivienda de Ana encontramos tres ordenadores, dos de ellos son portátiles y uno fijo. Uno de los portátiles pertenece al padre y es usado como herramienta para la gestión contable de su empresa. Otro portátil es propiedad de la hermana mayor, la cual también lo utiliza prioritariamente para su ámbito profesional, y el fijo que está disponible para todos los miembros de la unidad familiar. Poseen conexión a internet, un fax, teléfono, tres televisores con TDT y móviles para cada uno de los miembros de la familia. Igualmente, encontramos un equipo de música y una cámara de fotografía de propiedad exclusiva de Ana. Dispone también de un móvil cuya tarifa es de contrato.

En cuanto a la propiedad del ordenador, Ana manifiesta que lo comparte con sus hermanas, habiendo realizado una partición del disco duro para habilitar tres espacios de uso individual.

\subsection{Ecosistema tecnológico del centro educativo}

El Instituto al que asiste Ana es un centro público situado en un barrio de la periferia de la capital cuyo nivel económico y sociocultural es medio. Es un centro comprometido con la innovación y la mejora, cuyas señas de identidad se aprecian en los múltiples proyectos en los que participa: proyecto bilingüe, proyecto escuela espacio de paz, plan de lectura y biblioteca, proyecto de coeducación, proyecto Ecoescuela, Comenius Project, etc.

El Instituto de Ana es un centro TIC que inicia su andadura, como tal, el curso escolar 2005/06. Posee una página web en la que se puede encontrar abundante información del centro y de todas las actividades e iniciativas que se proponen y desarrollan en el mismo. 
El alumnado usa el ordenador en sus aulas ordinarias, aunque no todas ellas están equipadas. La asignación de las aulas TIC se ha hecho por departamentos, de forma análoga a las aulas normales, cuya distribución lleva experimentándose varios años con unos resultados excelentes, según manifiestan algunos docentes.

Es destacable la regulación de normas de uso de los medios digitales que se establecen en el Instituto. Se trata de unas normas públicas que, básicamente, hacen referencia a la supervisión del buen funcionamiento de los equipos.

\subsection{Inclusión tecnológica o cambio educativo. Esa sí es la cuestión.}

El primer interrogante que abordamos se centra en el impacto que tienen las TIC para transformar las prácticas educativas. En el estudio de caso comprobamos que el profesorado participante manifiesta que en los centros y aulas, parece existir una gran distancia entre el ámbito formal y burocrático, en el que se van cumpliendo satisfactoriamente objetivos (tales como la dotación de materiales, equipamientos y apoyos técnicos en los centros educativos), y el ámbito real y práctico que requiere superar resistencias del profesorado al uso de estos recursos en los procesos de enseñanza-aprendizaje, coincidiendo con la opinión mostrada por Angulo \& Vázquez (2010).

Ana manifiesta que el uso de TIC en el aula se realiza de forma muy limitada, controlada y poco interesante. Ella considera que, pese a ser un centro TIC, el uso del ordenador es muy escaso y hay una excesiva reducción de acceso a determinados contenidos:

A: "Sí. Bueno, el TIC, el ordenador se utiliza poco porque ninguno funciona pero, en fin..." (ER1).

A: "Casi todas las páginas están denegadas, o sea que no te puedes meter en casi...La única es la plataforma del Instituto y de ahí no sales" (ER1).

Ana indica que el uso prioritario atribuido al ordenador es la búsqueda de información, empleando como página favorita de consulta la Wikipedia. La búsqueda de información es, dentro de la competencia digital, una de las habilidades más básicas, de la que no deriva directamente un verdadero aprendizaje y adquisición de conocimiento. El profesorado, al usar TIC, se preocupa básicamente por controlar y evitar la copia de la información, desatendiendo o relegando a un segundo plano, funciones relativas a la transformación de la información en conocimiento.

Ana expresa su experiencia de aprendizaje con TIC del siguiente modo:

\section{E1: ¿Y los profes no os dicen nada si lo copiáis todo de la Wikipedia?}

A: "Bueno, sí nos dicen que no lo copiemos igual. Que tenemos que resumirlo... Ellos nos dicen que tenemos que presentarlo a mano para que no sea solo copiar, cortar e imprimir... Pero vamos que te cansas de copiar un montón de tiempo..." (EA1)

Por su parte, el vicedirector también muestra ciertas reservas respecto al nivel de formación en TIC del profesorado y a su inclusión real de estos medios en las prácticas de aula. Indica que su uso está muy vinculado al dominio personal de cada docente y al planteamiento metodológico que hace de su materia. Constata que existe una preocupación por parte de la administración y que se ha incorporado a nivel burocrático un apartado en las 
programaciones. El que se cumplimente este apartado no significa que se produzca una transferencia inmediata al aula. El vicedirector cree que se trata más de un reto de futuro que de una realidad presente. Lo indica del siguiente modo:

"Es un proceso que está por llegar digamos, eso ahora mismo es incipiente y depende de la iniciativa particular de algún profesorado que, concretamente, quiera pues..." (EP1).

El profesorado entrevistado es consciente de la limitación que supone un uso mecánico de las TIC, advirtiendo que los beneficios para su desarrollo dependerán de la calidad de la actividad propuesta y de los procesos mentales que active en el alumnado.

P: "Sí, a ver, las nuevas tecnologías no...intrínsecamente no tienen por qué verse como negativas, lo negativo es el uso que se les pueda dar o...es decir, o que su uso se plantee de tal manera que no signifique procesos mentales por parte del alumnado". (EP1).

Coincide su opinión con las aportaciones que ofrecen Pérez, Aguaded y Fandos (2010: 312) cuando expresan: "El profesorado percibe las TIC como recursos valiosos por su potencial didáctico y educativo, aunque afirman que el uso suele ser irregular, puntual y discontinuo".

En cualquier caso, el uso de TIC en el aula no garantiza un cambio en el modelo pedagógico del profesorado, como se corrobora en investigaciones sobre el tema (Buckingham, 2008; Area, 2010). Aunque utilicemos un 'barniz de modernidad' con nueva aparatología podemos estar ante un mismo modelo educativo centrado en la transmisión de información que esté muy lejos de favorecer la implicación y actividad del alumnado en la construcción de sus conocimientos. Por ello, hemos de tener en cuenta que la formación profesional de los docentes no se debe reducir exclusivamente a desarrollar habilidades tecnológicas, sino sobre todo, pensar en cómo incorporar las TIC dentro de un modelo activo y colaborativo de aprendizaje. Ello implica, necesariamente, la mejora de las competencias pedagógicas docentes.

En este sentido, desvincular el conocimiento técnico de las herramientas digitales de los fines educativos a los que ha de servir significa tecnificar los procesos de enseñanzaaprendizaje sin contribuir a un verdadero desarrollo de las competencias del alumnado.

\subsection{Historia de un desencuentro... Las competencias digitales desde la mirada de los protagonistas del aula}

Otros interrogantes que han marcado la exploración realizada en este estudio de caso es la percepción que tiene Ana y el profesorado entrevistado respecto al dominio de las competencias digitales.

Ana, al pertenecer a la generación net, se ha alfabetizado en las tecnologías digitales y presenta un dominio intenso de las habilidades técnicas, las cuales mejora día a día a través de un entrenamiento continuado, según el tiempo de uso que su madre y ella misma expresa:

M: "Ella haciendo sus tareas y estudiando lo mínimo...lo demás es todo ordenador".

A: Sí, muchas. En fines de semana me puedo tirar..." 
Y su madre apostilla:

M: "Todo el día, 8 ó 10 horas, sí. Se quita a lo mejor para ir al servicio, o ir a comer o merendar y son muchas horas. Yo le digo 'Ana, hay más vida fuera del ordenador', pero vamos que es lo que yo..." (EA1).

Fruto de esta familiarización con la tecnología provoca un nivel de seguridad elevado, lo cual la sitúa en una actitud de indagación, búsqueda y exploración permanente en la red. Tanto Ana como su madre, no consideran el centro escolar como espacio privilegiado para el aprendizaje del uso del ordenador, indicando que éste lo realiza de forma autodidacta -por ensayo y error-, a través de algunas directrices que le proporciona su hermana mayor y "lo poquillo que le enseñan en el colegio" (M).

En este proceso autodidacta los nuevos aprendizajes adquiridos se instauran en una estructura modular que facilita seguir profundizando en el dominio de las TIC, actuando como una espiral formativa compleja e indefinida en la que interactúan positivamente elementos motivacionales, de ocio y de socialización.

El uso de las TIC que realizan los jóvenes en contextos no formales contrasta con el uso sistemático, reducido y controlado que se realiza desde los escenarios de educación formal. La supervisión y control que ejerce el profesorado limita ostensiblemente sus posibilidades sobre todo de acceso a diversos contenidos.

Por su parte, el profesor entrevistado admite el dominio digital del alumnado pero identificándolo, casi en exclusividad, con el ámbito lúdico.

P: "Sí, Ana y en general todos los chicos y chicas de su edad dominan perfectamente los ordenadores. Es su mundo, se han criado con ellos... Les gustan, les divierten, les permiten acceder a lugares remotos, mostrarse no como son, sino como les gustaría ser... ¿qué mas quieres? Donde se ponga un buen ordenador, que se quiten todos los libros del mundo..." (EP1)

Al igual que Ana, el profesor entrevistado muestra su convicción sobre la falta de autorregulación de los y las adolescentes en el uso del ordenador en el contexto y para las finalidades académicas establecidas. Expresa que si no existiera una supervisión y control externo el alumnado se metería en las redes sociales y no trabajaría en el contenido disciplinar que se le propone.

P: "El problema es que casi siempre los utilizan para jugar, para chatear, para conectarse con los amigos a las redes sociales... Mucha diversión, pero aprender, lo que se dice aprender, poca cosa" (EP2)

El docente nos indica que, en la mayoría de los casos, el uso que se les propone a los y las adolescentes en el centro es la utilización del ordenador para que les ayude en la preparación de algún trabajo académico, vinculando su uso básicamente a búsqueda de información. No contemplan su utilidad como recurso de socialización o contacto con otros jóvenes a excepción de algún caso de programa de intercambio bilingüe.

Al igual que en el ecosistema familiar percibimos ciertos temores por parte del docente en relación al uso que hacen los jóvenes de las TIC. Él considera que ha de racionalizarse su uso, refiriéndose sobre todo al tiempo permitido. Advierte como hipotéticos peligros la falta de 
socialización en contextos reales de los chicos y chicas, así como un nivel poco deseable de dependencia respecto al núcleo familiar. Considera que puede resultar cómodo mantener relaciones virtuales desde un contexto seguro como puede ser el dormitorio de casa. Como contrapartida, advierte de la ralentización que esta conducta, empleada en exceso, puede generar respecto a la maduración y seguridad que van adquiriendo los y las adolescentes al participar en contextos sociales reales cada vez más amplios, complejos y desconocidos, alejados de la protección familiar.

P: "En casa tenemos que tener cuidado de poner límites porque si no, ellos estarían un montón de horas y no habría ni límite ni autocontrol del número de horas que podrían estar usando ese tipo de medios" (EP1).

Contrasta su opinión, respecto al necesario control que han de ejercer los progenitores en la limitación de los tiempos dedicados a las TIC, con la laxitud de normas temporales y de acceso a diversos contenidos que apreciamos en el entorno familiar de Ana. Podemos decir que en la familia de Ana se ha ejercido un traspaso de control del padre y de la madre a las hermanas mayores de Ana y, finalmente, es su propia autorregulación la que administra los tiempos y contenidos de acceso a las TIC. La percepción del profesor entrevistado es que la situación de nuestra protagonista no es una excepción respecto a la mayoría de las familias del centro.

\section{CONCLUSIONES}

Los resultados de este estudio muestran la persistencia de una concepción formal e institucional en el uso de las TIC en los contextos educativos, lo cual no hace mas que confirmar la permanencia de un modelo que ha sido el predominante en la anterior década y que dificulta una verdadera transformación de las prácticas pedagógicas. En esta situación inciden factores determinantes como los procesos de socialización del profesorado con las TIC y una extendida cultura pedagógica, propia de la escuela de la sociedad industrial, que impone criterios didácticos y de organización escolar que excluyen las virtualidades que pueden aportar las modernas tecnologías. Los datos recabados evidencian que dichos procesos de socialización son significativamente distintos en el caso del profesorado respecto al alumnado respecto a la funcionalidad y la manera de entender las relaciones del usuario con el ordenador y la red -interfaz de usuario según Manovich (2005)-.

Por otra parte, hemos constatado como entre determinados sectores de la población, con un limitado capital cultural, los procesos de socialización con los nuevos medios de comunicación asumen una concepción restringida de la denominada cultura de la convergencia (Jenkins, 2008), lo que provoca que los saberes y valores que ofrecen los progenitores a los y las adolescentes se asemejen a los del profesorado. En ambos casos se mantiene presencia del viejo paradigma "vacunador" ante los medios, junto con la insuficiente capacidad para educar en un nuevo modelo cultural caracterizado por nuevas formas de gestión, producción, difusión y consumo de la información y de relación en y con la red.

Del análisis de los factores que mantienen el que hemos denominado "choque cultural" en las aulas, destacamos como el predominio de la cultura letrada presente en la cultura profesional de los docentes, se concreta en un modelo de comunicación en el aula dirigido, propio de las aulas predigitales, en el que el control sobre la información ocupa un papel 
importante aunque en las prácticas educativas se estén utilizando las TIC. Esta afirmación es fácilmente constatable al analizar los modelos organizativos del aula, así como la estructuración de espacios y recursos tecnológicos. Mientras el alumnado, en los contextos extraescolares, hace un uso de los modernos medios como incipientes prosumidores, poniendo en práctica modelos basados en la participación e interactividad, tal y como la concretan Aparici y Silva (2012).

Otra de las evidencias derivadas de esta investigación son las percepciones mutuas que mantienen alumnado y profesorado sobre los niveles de competencia digital. Mientras que el alumnado tiene la percepción de que las habilidades tecnológicas de los docentes son escasas, el profesorado apunta que la competencia digital del alumnado se encuentra circunscrita a ciertos ámbitos relacionados con el ocio, mostrándose inmaduro para un uso racional del recurso. En ambos casos existe escaso interés y pocas habilidades que permitan buscar el punto de encuentro necesario y sus puntos de vista suelen ser considerados recíprocamente como inamovibles. Una de las posibilidades para avanzar en la solución de esta cuestión, que pone en evidencia otro aspecto de la brecha digital, es enmarcar las prácticas educativas dentro de los modelos dialógicos y comunitarios de aprendizaje (Segovia, 2010). Los diferentes modos de apropiación de la cultura de la información de adultos y adolescentes pueden ser complementarios buscando modelos de articulación más interactivos, propios de la red.

Tras las conclusiones apuntadas anteriormente, consideramos que la superación de este choque cultural, respecto a la integración y uso de las TIC en la enseñanza, requiere un modelo docente activo, crítico y colaborativo, en el que las posibles diferencias en cuanto a competencias digitales entre alumnado y profesorado se conciban como una oportunidad de formación y aprendizaje cooperativo. En este sentido, entendemos que se ha de promover la búsqueda de puntos de convergencia y encuentro entre las percepciones de docentes y estudiantes y que ambos entiendan y valoren las posibilidades las TIC, en el contexto de una necesaria alfabetización mediática, tanto en ámbito lúdico como en el educativo, lo que requiere de la integración de la cultura popular relacionada con los nuevos medios en la práctica curricular.

Igualmente es necesario delimitar las funciones que ha de asumir la institución escolar respecto a los procesos de alfabetización mediática, teniendo en cuenta las limitaciones tecnológicas a las que se encuentra sometida. Ello supone una ampliación de la concepción de la alfabetización letrada a la que tradicionalmente ha estado vinculada la escuela, por lo que es importante reformular los saberes curriculares relacionados con la alfabetización, los métodos de enseñanza, los recursos educativos y la organización de los espacios y tiempos escolares.

\section{REFERENCIAS BIBLIOGRÁFICAS}

ANGULO RASCO, J. F., \& VÁZQUEZ RECIO, R. (2010). El currículum y los nuevos espacios para aprender. In J. Gimeno Sacristán (Ed.), Saberes e incertidumbres sobre el currículum. (pp. 501-526) Madrid: Morata.

APARICI, R. \& SILVA. M. (2012). Pedagogía de la interactividad. Comunicar. 38, 51-58. 
AREA, M. (2010). El proceso de integración y uso pedagógico de las TIC en los centros educativos. Un estudio de casos. Revista de Educación, 77-97.

AREA, M. (coord.) (2011). Estudio 1: ¿Qué opina el profesorado sobre el Programa Escuela 2.0? Un análisis por comunidades autónomas (Avance de resultados). http://www.ite.educacion.es/w3/3congresoe20/Informe_Escuela20-Prof2011.pdf

BALANSKAT, A., BLAMIRE, R., \& KEFALA, S. (2006). The ITC impact on schools in Europe. (http://ec.europa.eu/education/pdf/doc254 en.pdf) (18-1-2011)

Bolívar, A. (Comp.) (2007). Análisis del discurso. Por qué y para qué. Colección Minerva. Manuales Universitarios. Venezuela: Universidad Central de Venezuela.

BUCKINGHAM, D. (2008). Más allá de la tecnología. Aprendizaje infantil en la era de la cultura digital. Buenos Aires: Manantial.

CLANDININ, J. \& CONNELLY, M. (2000). Narrative Inquiry: Experience and Story in Qualitative Research. San Francisco, CA: Jossey-Bass.

COLL, C. (2008). Aprender y enseñar con las TIC. Expectativas, realidad y potencialidades. Boletín de la Institución Libre de Enseñanza, 72, 17-40.

COLLER, X. (2000). Estudio de casos. (Cuadernos metodológicos, 30). Madrid: C.I.S.

CONDIE, R., \& MUNRO, B. (2007). The impact of ICT in European Schools - a landscape review. In BECTA (Eds.) (http://dera.ioe.ac.uk/1627/)

DENZIN, N. K., LINCOLN, Y. S. (1994). Introduction: Entering the Field of Qualitative Research y The Fifth Moment. En N. H. Denzin \&Y. S. Lincoln (eds.), Handbook of Qualitative Research. California: Sage Publications.

EISNER, E. W. (1998). El ojo ilustrado. Indagación cualitativa y mejora de la práctica educativa. Barcelona: Paidós.

FARÍAS, L., \& MONTERO, M. (2005). De la transcripción y otros aspectos artesanales de la investigación cualitativa. International Journal of Qualitative Methods, 4 (1), Article 4. http://www.ualberta.ca/ iiqm/backissues/4_1/pdf/fariasmontero.pdf

HUBERMAN, M. (1998): Trabajando con narrativas biográficas. En H. McEwan \& K. Egan (Comps.), La narrativa en la enseñanza, el aprendizaje y la investigación, 183-235. Buenos Aires: Amorrortu.

Instituto Nacional de Tecnologías de la Comunicación (2009). Estudio sobre hábitos seguros en el uso de las TIC por niños y adolescentes y e-confianza de sus padres. http://www.inteco.es/Seguridad/Observatorio/Estudios e Informes/Estudios e Inf ormes $1 /$ Estudio ninos

JENKINS, H. (2008). Convergente Culture. Barcelona: Paidós.

LANKSHEAR, C., \& KNOBEL, M. (2008). Nuevos alfabetismos: su práctica cotidiana y el aprendizaje en el aula. Madrid: Morata.

MANOVICH, L. (2005). El lenguaje de los nuevos medios de comuicación. Barcelona: Paidós. 
PÉREZ, M.A., AGUADED, I. \& FANDOS, M. (2010). Percepciones, retos y demandas de los directores y coordinadores de los centros TIC andaluces. Educatio Siglo XXI, 28 (1), 295-316.

SEGOVIA, B. (2010). Educación comunitaria y nuevas alfabetizaciones. En R. Aparici (coord.) Conectados en el ciberespacio. Madrid: UNED.

SIGALÉS, C., MOMINÓ, J. M., \& Meneses, J. (2007). La escuela en la sociedad red. Internet en la educación primaria y secundaria. Barcelona: Ariel.

STAKE, R. E.(1998). Investigación con estudio de casos. Madrid: Morata.

VAN DIJK, T. (Ed.) (2001). El discurso como estructura y proceso. Estudios sobre el discurso I. Una introducción multidisciplinaria. Madrid: Gedisa.

\section{Para citar este artículo:}

SEGOVIA, B., MÉRIDA, R., GONZÁLEZ, E. \& OLIVARES, M.A. (2013). Choque cultural en las aulas: profesores analógicos vs alumnado digital. El caso de Ana. EDUTEC, Revista Electrónica de Tecnología Educativa, 43 Recuperado el $\mathrm{dd} / \mathrm{mm} / \mathrm{aa}$ de http://edutec.rediris.es/Revelec2/Revelec43/choque cultural aulas profesores analogicos alumnado digital.html 